\title{
Cleaving plane-dependent electronic structures of transition metal diarsenides
}

\author{
Gyanendra Dhakal, ${ }^{1}$ M. Mofazzel Hosen, ${ }^{1}$ Wei-Chi Chiu, ${ }^{2}$ Bahadur Singh, ${ }^{3}$ Cheng-Yi Huang, ${ }^{2}$ Klauss Dimitri, ${ }^{1}$ \\ Baokai Wang, ${ }^{2}$ Firoza Kabir, ${ }^{1}$ Christopher Sims, ${ }^{1}$ Sabin Regmi $\odot,{ }^{1}$ William Neff, ${ }^{1}$ Jonathan Denlinger, ${ }^{4}$ Hsin Lin, ${ }^{5}$ \\ Dariusz Kaczorowski $\odot,{ }^{6}$ Arun Bansil, ${ }^{2}$ and Madhab Neupane ${ }^{1}{ }^{1, *}$ \\ ${ }^{1}$ Department of Physics, University of Central Florida, Orlando, Florida 32816, USA \\ ${ }^{2}$ Department of Physics, Northeastern University, Boston, Massachusetts 02115, USA \\ ${ }^{3}$ Department of Condensed Matter Physics and Materials Science, Tata Institute of Fundamental Research, Mumbai 400005, India \\ ${ }^{4}$ Advanced Light Source, Lawrence Berkeley National Laboratory, Berkeley, California 94720, USA. \\ ${ }^{5}$ Institute of Physics, Academia Sinica, Taipei 11529, Taiwan \\ ${ }^{6}$ Institute of Low Temperature and Structure Research, Polish Academy of Sciences, Okolna 2, 50-422 Wroclaw, Poland
}

(Received 16 January 2020; accepted 21 April 2021; published 2 June 2021)

\begin{abstract}
Topological Dirac and Weyl semimetals are currently attracting intense interest due to their exotic physical properties. Transition metal diarsenides such as $\mathrm{MoAs}_{2}$ and $\mathrm{WAs}_{2}$ have been reported to harbor very high magnetoresistance suggesting the possible existence of a topological quantum state, although this conclusion remains dubious. Here, using systematic angle-resolved photoemission spectroscopy (ARPES) measurements and parallel first-principles calculations, we discuss the electronic structures of $\mathrm{TAs}_{2}(\mathrm{~T}=\mathrm{Mo}, \mathrm{W})$. Two different cleavage planes of $\mathrm{MoAs}_{2}$ are found to harbor distinctly different surface states. Our experiments show the presence of Dirac-like dispersions on the (001) plane, which our first-principles calculations ascribe to trivial surface states. Our in-depth study also finds $\mathrm{WAs}_{2}$ to possess a trivial electronic structure. Our study emphasizes the importance of identifying the cleavage plane in low-symmetry systems and indicates that topological semimetallic states are not the key for generating high magnetoresistance in $\mathrm{MoAs}_{2}$ and $\mathrm{WAs}_{2}$.
\end{abstract}

DOI: 10.1103/PhysRevResearch.3.023170

\section{INTRODUCTION}

Experimental discovery of a 3D topological insulator state is widely acknowledged as a major milestone in condensed matter and materials physics [1-5]. This discovery has spurred intense research interest in other classes of topological materials, and exotic states such as the Dirac semimetal [6-12], Weyl semimetal [13-19], and nodal-line semimetal [20-23] have been theoretically proposed and experimentally realized. In Dirac semimetals, linearly-dispersing bands cross at low energies and behave like Dirac fermions. Since inversion and time-reversal symmetries protect the Dirac states, their realization requires the material to be centro-symmetric and nonmagnetic [24,25]. Breaking either the inversion or the time-reversal symmetry drives a Dirac semimetal into a Weyl semimetal. Weyl fermions evolve as low-energy excitations at topologically protected crossing points (Weyl nodes) between the electron and hole bands. Weyl nodes act as monopoles or antimonopoles of Berry curvature depending on their chirality. Pairs of nodes of opposite chiralities are separated in momentum space and behave like magnetic dipoles [13,24,25]. A recent player on the scene is a type-II Weyl semimetal, which

\footnotetext{
*madhab.neupane@ucf.edu
}

Published by the American Physical Society under the terms of the Creative Commons Attribution 4.0 International license. Further distribution of this work must maintain attribution to the author(s) and the published article's title, journal citation, and DOI. does not conserve Lorentz invariance in that its Weyl cones are tilted and the electron- and hole-like pockets intersect at the Fermi surface [26]. Open Fermi surfaces, anisotropic chiralities, and unconventional anomalous Hall effect are among the unusual features of type-II Weyl semimetals. Type-II Weyl fermions are often associated with gigantic magnetoresistance [24,25]. ARPES studies along with parallel first-principles calculations on $\mathrm{MoTe}_{2}, \mathrm{WTe}_{2}, \mathrm{MoP}_{2}$, and $\mathrm{WP}_{2}$ [27-30] confirm their type-II Weyl characteristics. These advances have spurred the exploration of type-II Weyl states in other binary compounds of Mo and W. Transition metal diarsenides have drawn special attention due to their high magnetoresistance and carrier density-of-states [31].

Recent reports of topological properties and the origin of high magnetoresistance in $\mathrm{MoAs}_{2}$ present contradictory results [32-34]. A first-principles calculation finds states with nontrivial $\mathbb{Z}_{2}$ index and suggests high magnetoresistance to result from electron-hole compensation [32]. However, another study reports trivial band structures and argues that the high magnetoresistance results from carrier motion on the Fermi surface, which is enriched via the open-orbit topology [33]. A quantum oscillation experiment complemented by first-principles calculations indicates the existence of open-orbits in $\mathrm{MoAs}_{2}$ [34]. The need for a comprehensive experimental and theoretical analysis of the electronic structure of $\mathrm{MoAs}_{2}$ and its sister compound $\mathrm{WAs}_{2}$ is thus clear. A new level of understanding of the electronic structures can also be expected through the delineation of the termination dependence of the electronic structure that has not yet been reported. To date, ARPES measurements have also not been 
(a)

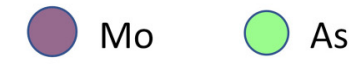

(b)

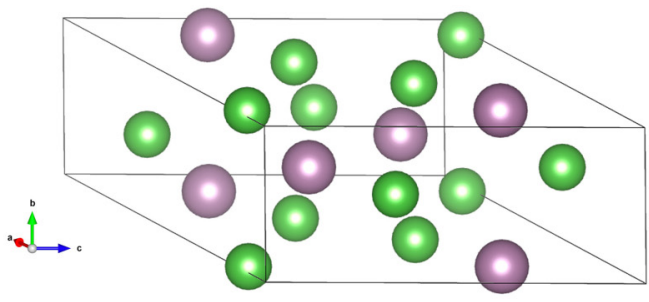

(d)

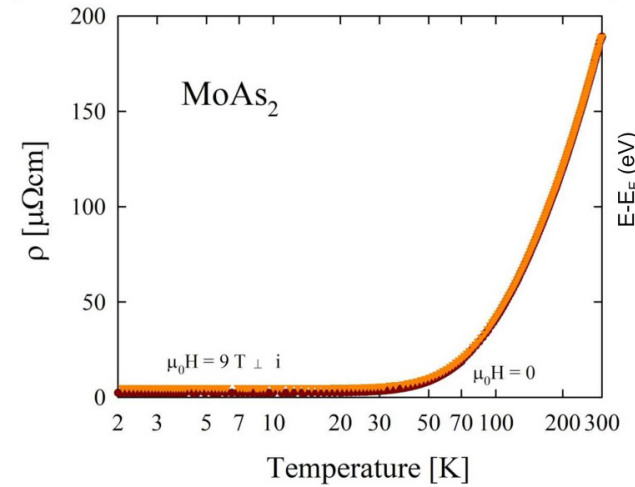

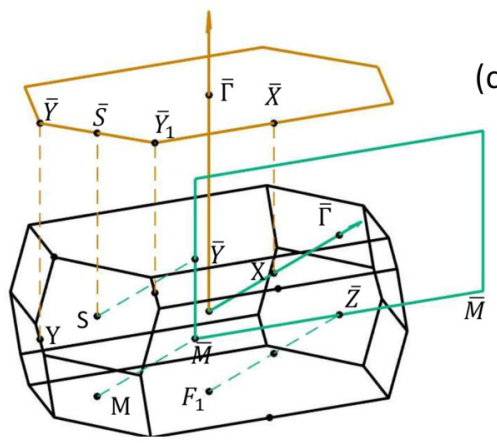

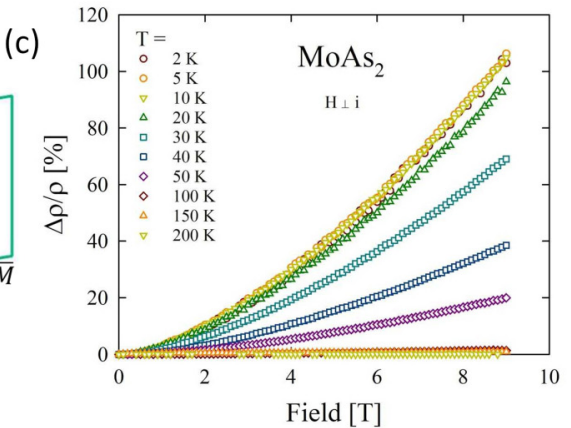

(e)

(f)
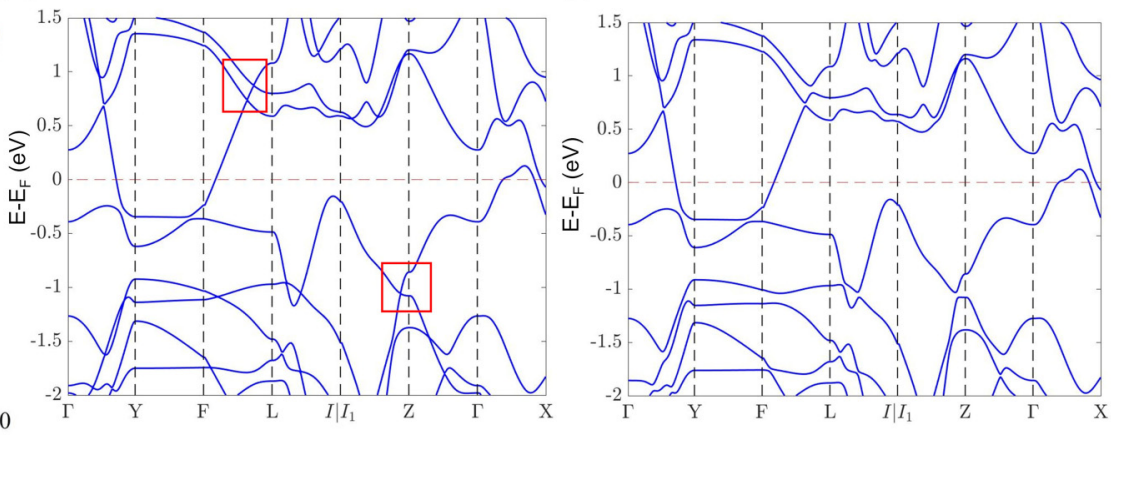

FIG. 1. Crystal structure, electronic transport, and bulk band structure of MoAs 2 . (a) Conventional unit cell of MoAs 2 . Purple and green spheres refer to Mo and As atoms, respectively. (b) Conventional 3D Brillouin zone of MoAs 2 with high-symmetry points labeled. Orange and green lines represent the conventional (001) and (100) surface projection, respectively. (c) Transverse magnetoresistance isotherms taken at different temperatures. (d) Resistivity measured as a function of temperature in zero field (dark red colored symbols) and in a field of 9 $\mathrm{T}$ applied perpendicular to current (orange-colored symbols). (e) Bulk band structure of MoAs 2 without SOC. Linearly crossed bands are enclosed in the red rectangles. (f) Bulk band structure of $\mathrm{MoAs}_{2}$ with SOC.

reported on the related compound $\mathrm{WAs}_{2}$, which belongs to the same crystal group as $\mathrm{MoAs}_{2}$ but with a stronger spin-orbit coupling (SOC) strength.

Here we report a systematic ARPES study of $\mathrm{TAs}_{2}$ $(\mathrm{T}=\mathrm{Mo}, \mathrm{W})$ with parallel theoretical calculations. Electronic structure of $\mathrm{MoAs}_{2}$ is delineated in two different cleavage planes. Both surfaces demonstrate the existence of trivial surface states. Importantly, the Dirac-like dispersions seen on the (001) surface are formed by trivial bands. We also reveal the presence of high magnetoresistance and similar Fermi surface features on the (001) surface of $\mathrm{WAs}_{2}$. Since $\mathrm{MoAs}_{2}$ and $\mathrm{WAs}_{2}$ both exhibit metallic band structures at low temperatures with highly populated trivial metallic surface states, our study indicates that topological semimetallic states are not the key for generating high magnetoresistance in these materials.

\section{METHODS}

\section{A. Sample growth and characterization}

The single crystals of $\mathrm{MoAs}_{2}$ and $\mathrm{WAs}_{2}$ were grown using the chemical vapor transport method with iodine as transporting agent [35]. The crystals formed needlelike structures with dimensions $\sim 1 \times 0.8 \times 0.4 \mathrm{~mm}^{3}$. The chemical composition was proven by energy-dispersive $x$-ray analysis using an FEI scanning-electron microscope equipped with an EDAX Genesis XM4 spectrometer. The crystal structure was examined at room temperature on a Kuma-Diffraction KM4 four-circle X- ray diffractometer equipped with a CCD camera using Mo $\mathrm{K} \alpha$ radiation. The experiment confirmed the monoclinic crystal structure and the lattice constants in accord with the previously reported values [35]. Electrical resistivity measurements were performed over 2 to $300 \mathrm{~K}$ employing a conventional four-point ac technique implemented in a Quantum Design PPMS platform. Electrical contacts were made using silver epoxy paste. A perpendicular magnetic field was applied to measure the transverse magnetoresistance.

\section{B. Spectroscopic surface measurements}

Synchrotron-based ARPES measurements were performed at the Advanced Light Source (ALS), Berkeley, at beamlines 4.0.3, 7.0.2, and 10.0.1 equipped with high-efficiency R8000 and R4000 electron analyzers. The energy resolution was set to be better than $20 \mathrm{meV}$. The angular resolution was set to be better than $0.2^{\circ}$ for all measurements. Samples were cleaved in situ and measured at 15 to $25 \mathrm{~K}$ in a vacuum better than $10^{-10}$ Torr. They were found to be very stable and without degradation for the typical measurement period of 20 hours. LEED measurements for structural analysis were performed at the ALS beamline 4.0.3 at a base pressure of better than $10^{-10}$ Torr in the preparation chamber. Samples were cleaved in the main chamber at a temperature of $80 \mathrm{~K}$ and moved to the preparation chamber for room-temperature LEED. The sample was further characterized by Laue diffraction. 


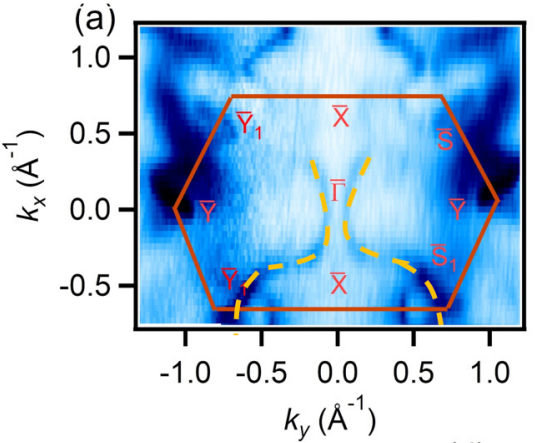

(c) $\bar{Y} \longleftarrow \bar{\Gamma} \longrightarrow \bar{Y}$

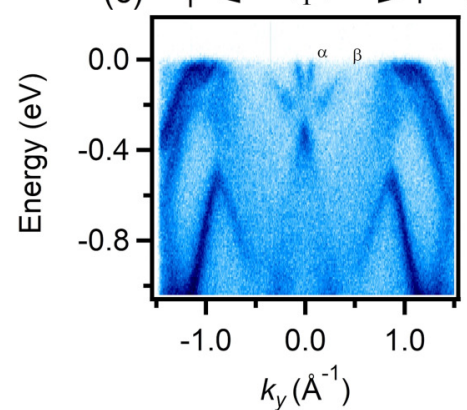

(b)

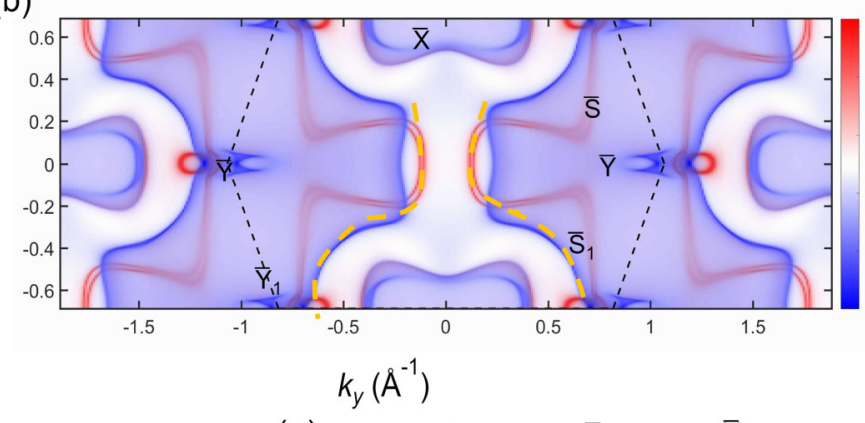

(e)
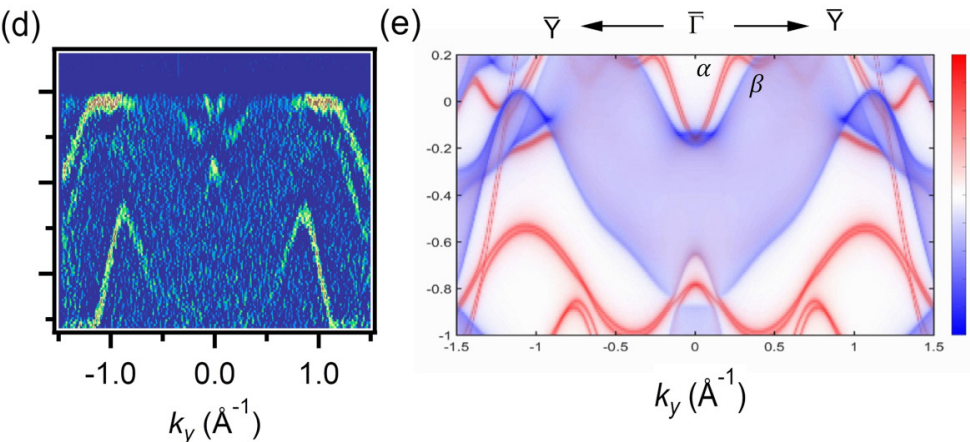

FIG. 2. Electronic structure of $\mathrm{MoAs}_{2}$ in the (001) plane. (a) Experimental Fermi surface map where high-symmetry points of the Brillouin zone are marked. Red lines represent the constructed Brillouin zone. The map was obtained using photon energy of $90 \mathrm{eV}$. (b) Calculated Fermi map. (c) Dispersion measurements along the $\bar{Y}-\bar{\Gamma}-\bar{Y}$ direction obtained at a photon energy of $98 \mathrm{eV}$. (d) Second-derivative plot of dispersion map in (c). (e) Calculated dispersion map along the $\bar{Y}-\bar{\Gamma}-\bar{Y}$ direction. All data were collected at the ALS beamline 4.0.3 at a temperature of $20 \mathrm{~K}$. The orange dotted curves are guides to the eye to compare theory and experimental plots. The blue color represents the bulk band whereas the red color denotes the surface band in the calculations.
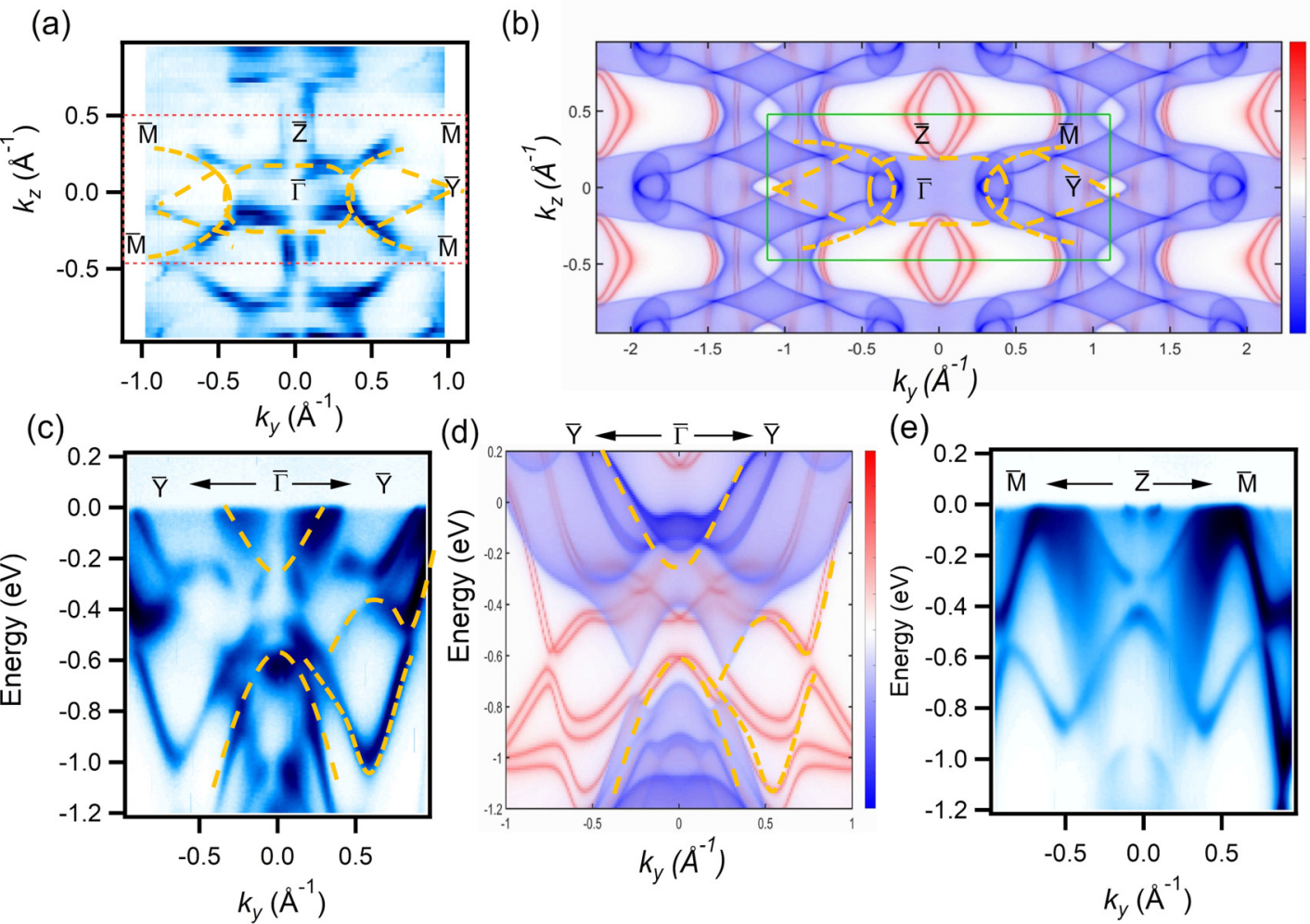

FIG. 3. Electronic structure of $\mathrm{MoAs}_{2}$ (100) surface. (a) Measured Fermi surface map of the MoAs 2 (100) plane. Dashed black lines represent the constructed Brillouin zone. (b) Calculated Fermi surface map. (c) Electronic band dispersion along the $\bar{Y}-\bar{\Gamma}-\bar{Y}$ direction. (d) Calculated dispersion map along the $\bar{Y}-\bar{\Gamma}-\bar{Y}$ direction. (e) Electronic band dispersion along the $\bar{M}-\bar{Z}-\bar{M}$ direction. Data were collected at the ALS beamline 10.0.1 at a temperature of $15 \mathrm{~K}$ using $70 \mathrm{eV}$ photon energy. The orange dotted curves serve as guides to the eye to compare theory and experimental plots. Blue color denotes surface state and red color represent the bulk state in the calculations. 
(a)

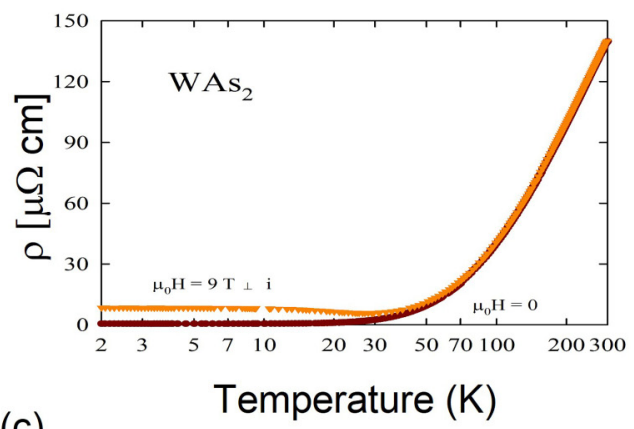

(c)

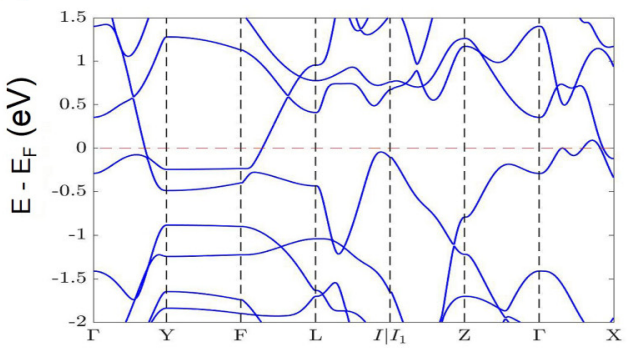

(b)

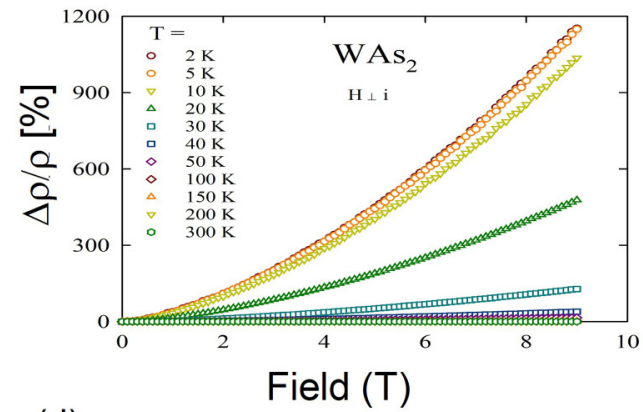

(d)

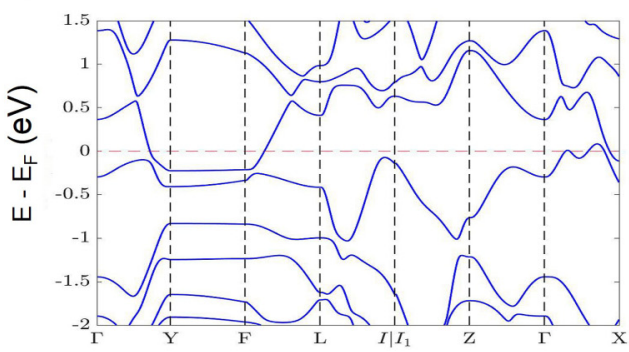

(e)

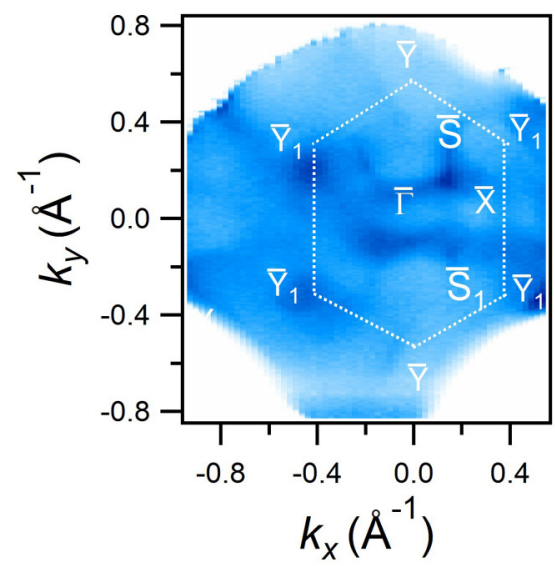

(f)

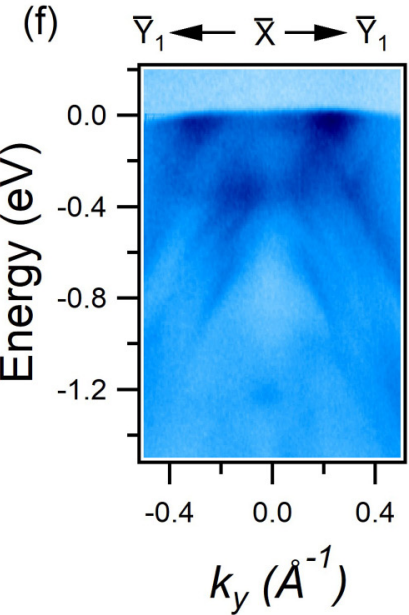

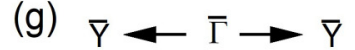

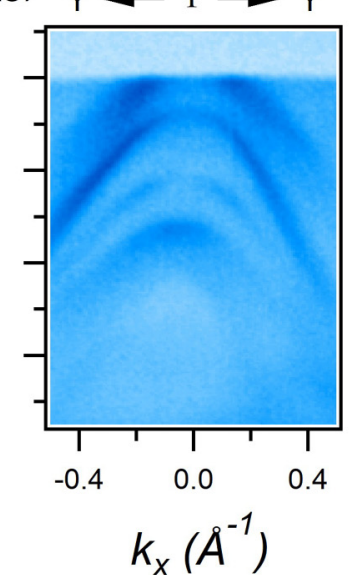

FIG. 4. Transport properties and the electronic structure of $\mathrm{WAs}_{2}$ of the (001) plane. (a) Resistivity as a function of temperature measured in zero field (dark red curve) and in a transverse field of $9 \mathrm{~T}$ (orange curve). (b) Transverse magnetoresistance isotherms measured as a function of the field at different temperatures. (c) Bulk band structure of WAs $s_{2}$ without SOC. (d) Bulk band structure of WAs $s_{2}$ with SOC. (e) Measured Fermi surface map of WAs 2 at the photon energy of $35 \mathrm{eV}$. (f) Energy-momentum dispersion of WAs $\mathrm{W}_{2}$ along the $\bar{Y}_{1}-\bar{X}-\bar{Y}_{1}$ direction. (g) Energy-momentum dispersion of $\mathrm{WAs}_{2}$ along the $\bar{Y}-\bar{\Gamma}-\bar{Y}$ direction. All data were collected at the ALS beamline 7.0.2 at a temperature of $20 \mathrm{~K}$.

\section{First-principles calculations}

Electronic structure was calculated by using the Vienna $a b$ initio simulation package (VASP) [36] based on the density-functional theory (DFT) [37] with the projector augmented wave (PAW) method [36,38]. We used the generalized-gradient approximation (GGA) [39] to model exchange-correlation effects. The energy cutoff of the plane wave basis set was $400 \mathrm{eV}$, and the size of the $k$ mesh was $16 \times 16 \times 7$. An effective tight-binding model Hamiltonian was obtained by projecting first-principles results onto Wannier orbitals using the VASP2WANNIER90 [40]. The Mo $d$ and As $p$ orbitals were included to generate Wannier func- tions. We calculated the surface states by using an iterative Green's function method with the WannierTools package [41].

\section{RESULTS AND ANALYSIS}

\section{A. Crystal structure and sample characterization}

$\mathrm{MoAs}_{2}$ crystallizes in a monoclinic structure in the $\mathrm{C} 2 / \mathrm{m}$ symmetry group (\#12) with lattice parameters: $\mathrm{a}=9.064(7)$ $\AA, \mathrm{b}=3.987(1) \AA, \mathrm{c}=7.7182(9) \AA$, and $\beta=119.37(1)^{\circ}$ [31,35], see Fig. 1(a). The conventional bulk Brillouin zone and its 2D projections onto the (100) and (001) surfaces are shown in Fig. 1(b). The temperature dependence of the 


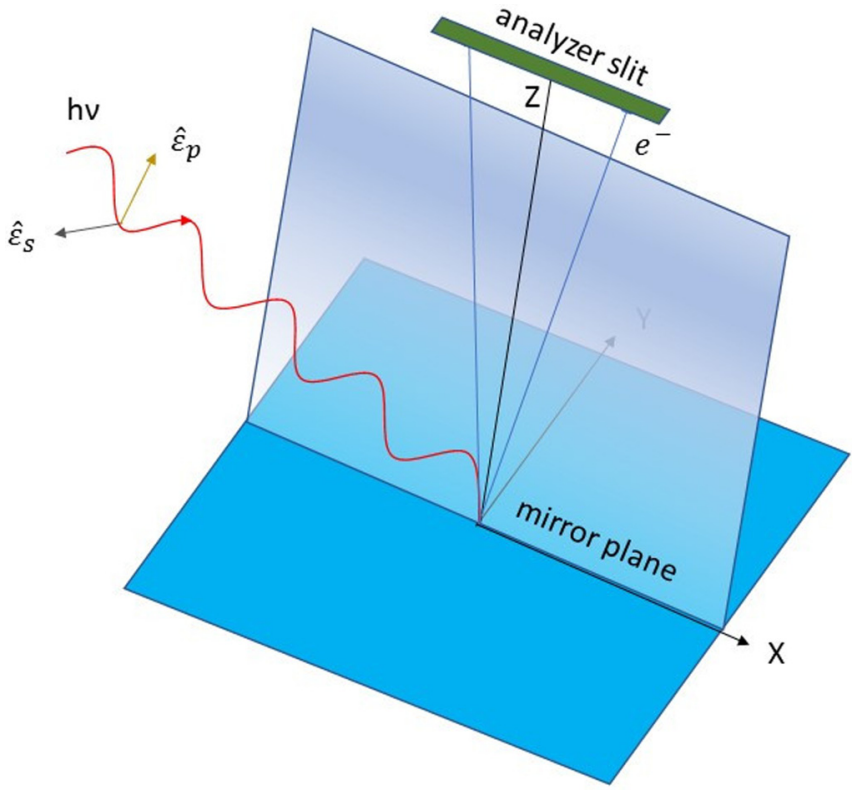

FIG. 5. Experimental geometry for ARPES measurements.

electrical resistivity of $\mathrm{MoAs}_{2}$ is presented in Fig. 1(d). At $300 \mathrm{~K}$, the resistivity is about $190 \mu \Omega \mathrm{cm}$ and decreases down to $2.6 \mu \Omega \mathrm{cm}$ at $2 \mathrm{~K}$. The large residual resistivity ratio $\left(\mathrm{RRR}=\rho_{300 K} / \rho_{2 K}\right)$ of 79 verifies high quality of the crystal measured. As seen in Fig. 1(d), magnetic field of $9 \mathrm{~T}$ applied perpendicular to electric current hardly alters $\rho(T)$. The transverse magnetoresistance (MR) taken as a function of the field at various temperatures from the range $2-200 \mathrm{~K}$ is presented in Fig. 1(c). At low temperatures, the magnitude of $\mathrm{MR}$ is enhanced $\left(\mathrm{MR}=107 \%\right.$ at $T=2 \mathrm{~K}$ and $\left.\mu_{0} H=9 \mathrm{~T}\right)$, and thus signals the possibility of $\mathrm{MoAs}_{2}$ hosting topological states and/or harboring electron-hole compensation. Moreover, the observed deviation of MR from the Kohler's rule can be associated with the behavior in a multiband system with variable carrier densities [31]. Bulk band structure of $\mathrm{MoAs}_{2}$ without and with the inclusion of spin-orbit coupling effects is presented in Figs. 1(e) and 1(f), respectively. The bands in the vicinity of the Fermi level give an impression of the Dirac dispersion in the regions enclosed in red rectangles $(0.8 \mathrm{eV}$ above the Fermi level near $\mathrm{L}$ and $1 \mathrm{eV}$ below the Fermi level near the $\mathrm{Z}$ point). These Dirac-like crossings are seen to take

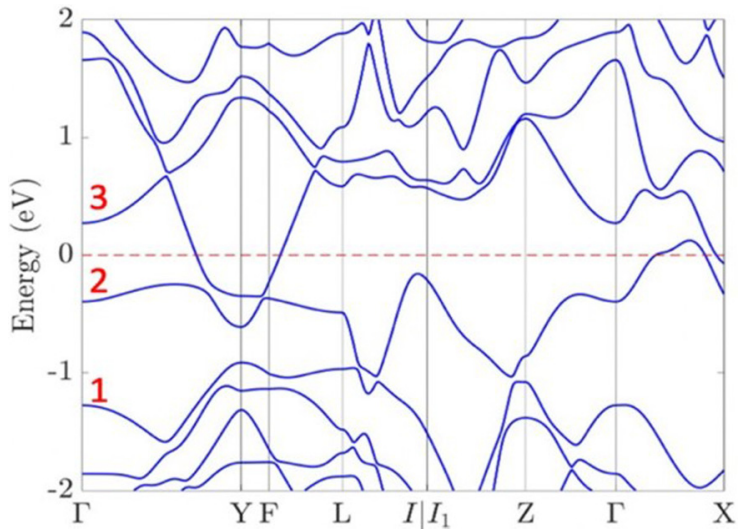

FIG. 7. Bulk-band structure of $\mathrm{MoAs}_{2}$ with spin-orbit coupling. Topological invariants were calculated by artificially shifting the Fermi level (dashed red line) to lie between bands 2 and 3.

place away from the high-symmetry points. Note that these Dirac-like crossings are not robust against spin-orbit coupling as gaps are opened up when spin-orbit coupling effects are included in the computations. Our $\mathbb{Z}_{2}$ calculations on MoAs show that it is topologically trivial with $\mathbb{Z}_{2}=(0 ; 000)$ when the Fermi level lies between the bands 2 and 3. It can, however, support a weak topological state with $\mathbb{Z}_{2}=(0 ; 111)$ if the Fermi level is artificially placed to lie between bands 1 and 2, see Appendix C for details.

\section{B. Observation of trivial band structures on the (001) plane}

We carried out ARPES measurements using a VUV photon source which allows surface-sensitive measurements. Electronic structures of the $\mathrm{MoAs}_{2}$ (001) surface are presented in Fig. 2. The experimental Fermi surface in Fig. 2(a) consists of multiple Fermi pockets indicating the metallic nature of $\mathrm{MoAs}_{2}$. A bridge-like pair of lines at the $\Gamma$ point is related to the surface bands. The experimental Fermi surface is reasonably captured by our first-principles calculations [Fig. 2(b)], some discrepancies can be attributed to matrixelements effects [42,43]. We draw orange-colored overlay plot to directly compare theory and experiment. Figure 2(c) displays the dispersion along the $\bar{Y}-\bar{\Gamma}-\bar{Y}$ direction. The inner linear band marked by $\alpha$, which disperses linearly does not change shape and size with photon energy, and manifests a (a)

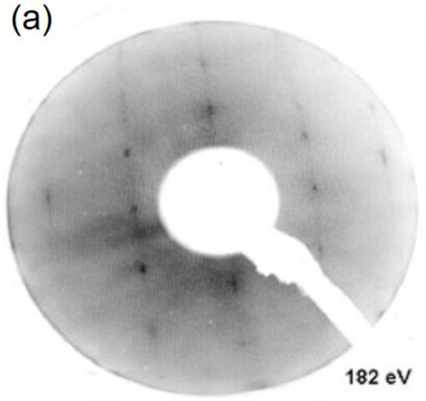

(b)

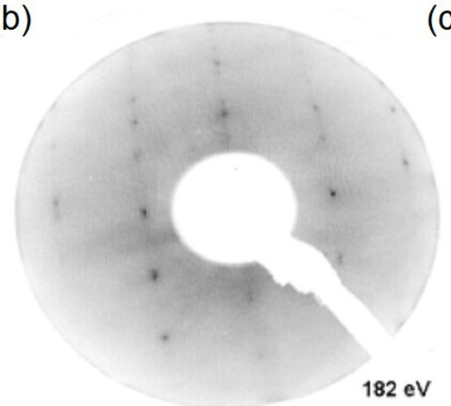

(c)

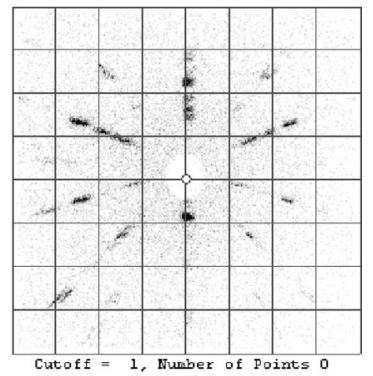

FIG. 6. LEED and Laue measurements. (a) LEED pattern of cleaved surface 1. (b) LEED pattern of cleaved surface 2. (c) Laue diffraction pattern of natural flat surface. 

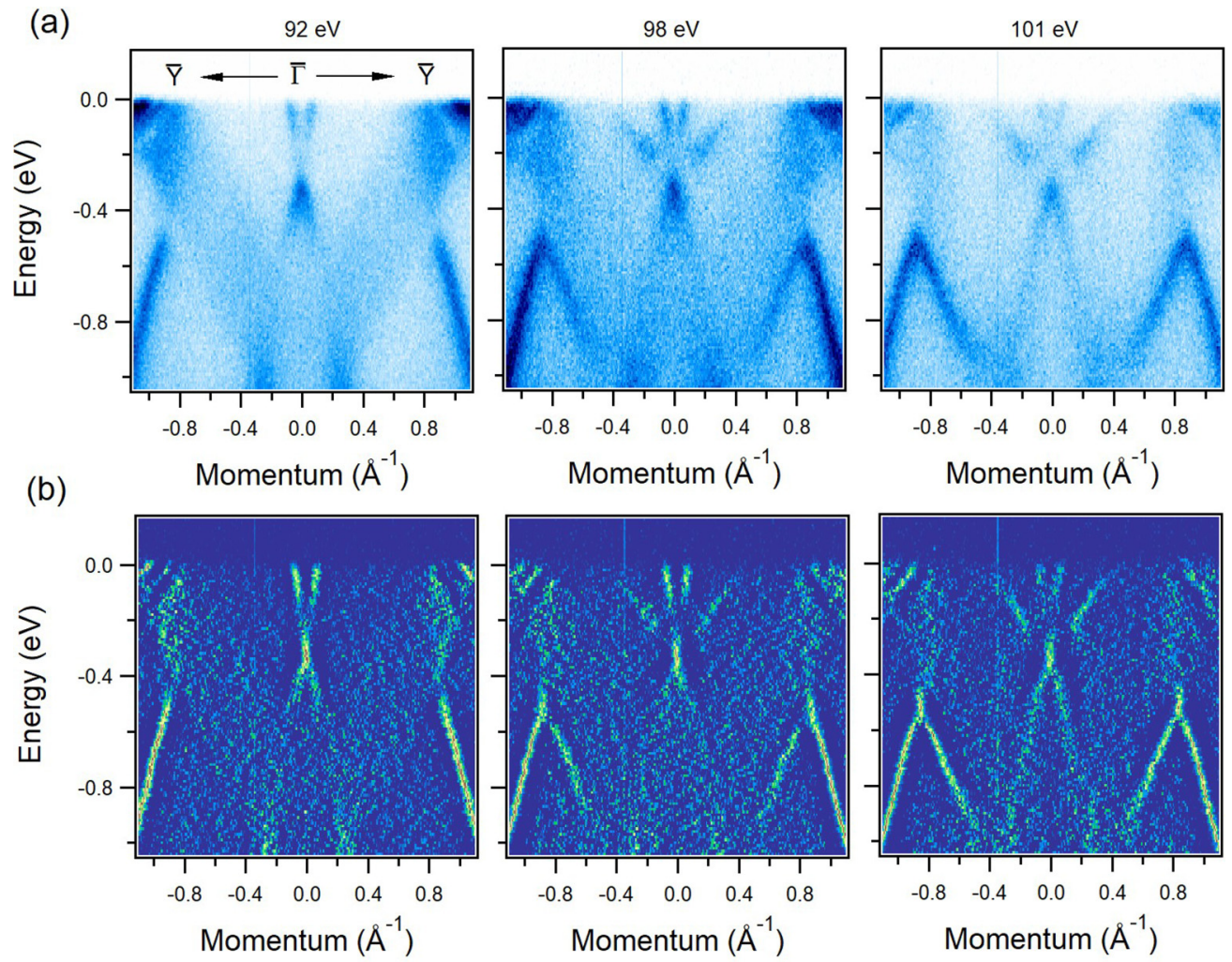

FIG. 8. Photon-energy dependent electronic dispersions and their second derivative plots along the $\bar{Y}-\bar{\Gamma}-\bar{Y}$ direction on the (001) surface in $\mathrm{MoAs}_{2}$. (a) Dispersion maps along the $\bar{Y}-\bar{\Gamma}-\bar{Y}$ direction for different photon energies as noted on the plots. (b) Second derivative plots corresponding to the maps presented in panel (a). All data were collected at the ALS beamline 4.0.3. at a temperature of $20 \mathrm{~K}$.

surface-originated Dirac-like dispersion. The $\alpha$-band is seen surrounded by the electron-like band marked $\beta$, which becomes stronger with varying photon energy, indicating its bulk nature. In particular, we do not see the presence of this bulk band in the Fermi surface map measured at a photon energy of $90 \mathrm{eV}$. Figure 2(d) shows a second-derivative plot of the map of Fig. 2(c) which highlights the linear crossings of the bands. In Fig. 2(e), the calculated dispersion map is presented. It reasonably captures the main bands observed in the experiment. Our theoretical calculations support our view that the $\alpha$ is a surface band and $\beta$ is a bulk band. Lower part of the $\alpha$ bands are not seen as they are buried in the bulk bands.

\section{Electronic structure of the (100) plane}

We now consider the surface-dependent electronic structure of $\mathrm{MoAs}_{2}$. Figure 3(a) shows that the Fermi surface map for the (100) plane is distinctly different from that for the (001) plane discussed in the preceding section. Approximately circular pockets are separated by a pair of lines. Extended

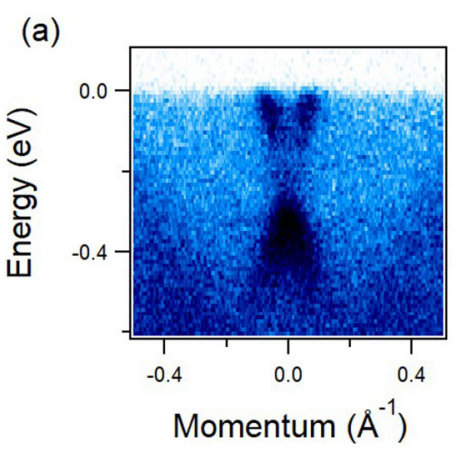

(b)

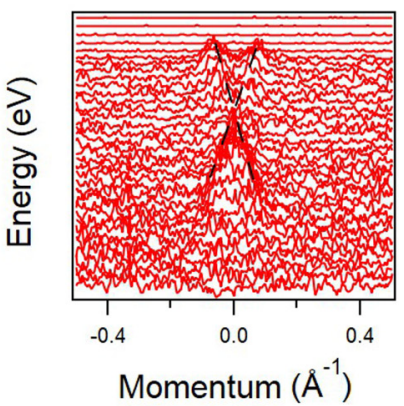

(c)

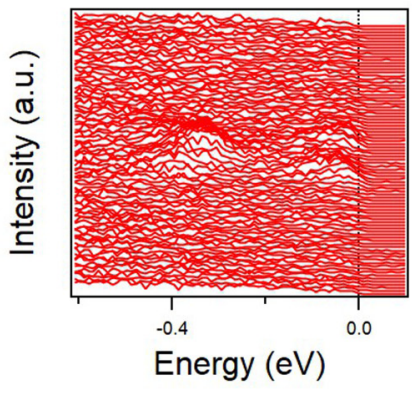

FIG. 9. ARPES measured (a) Dispersion map, (b) MDCs, and (c) EDCs along the $\bar{Y}-\bar{\Gamma}-\bar{Y}$ direction on the (001) surface. The data were taken at $20 \mathrm{~K}$ with photon energy of $92 \mathrm{eV}$. The dotted lines on the MDCs are guides to the eye. 
Fermi map here consists of multiple Brillouin zones presents a dumbbell shape. The rod-like feature along the $\bar{\Gamma}-\bar{Z}$ direction corresponds to electron-like bands. The calculated Fermi surface is presented in Fig. 3(b) and reasonably matches the experimental Fermi surface. Our data does not resolve all the features seen in the theoretical plot due presumably to the effects of experimental resolution and the ARPES matrix element. Figure 3(c) shows the dispersion along the $\bar{Y}-\bar{\Gamma}-\bar{Y}$ where multiple bands can be seen in the vicinity of $\bar{\Gamma}$ that cross the Fermi level. In Fig. 3(d), the first-principles calculations along the $\bar{\Gamma}-\bar{Y}$ direction demonstrate the surface states, which however are topologically trivial. Similarly, in Fig. 3(e), a pair of electron pockets can be seen in the vicinity of the $\bar{Z}$ point. A hole-like band appears at a higher binding energy and does not touch the electron pocket, an obvious gap can be seen. Interestingly, the gapless Dirac-like states are not seen in Figs. 3(c) and 3(e) (see Appendix D for details).

\section{Electronic structure of WAs}

$\mathrm{WAs}_{2}$ allows us to gain insight into the effects of tuning the strength of spin-orbit coupling in the transition-metal diarsenides by substituting Mo with W. Figure 4 shows the transport behavior and the detailed electronic band structures for the (001) surface of $\mathrm{WAs}_{2}$. The behavior of zero-field $\rho(T)$ and transverse MR is rather similar to that in $\mathrm{MoAs}_{2}$ [see Fig. 4(a) and 4(b)]. However, RRR of $\mathrm{WAs}_{2}$ is as large as 200 $\left(\rho_{300 \mathrm{~K}}=140 \mu \Omega \mathrm{cm}, \rho_{2 \mathrm{~K}}=0.7 \mu \Omega \mathrm{cm}\right)$, and MR measured at $\mathrm{T}=2 \mathrm{~K}$ and $\mu_{0} H=9 \mathrm{~T}$ reaches nearly $1200 \%$. As can be inferred from Fig. 4(a), in a transverse field of $9 \mathrm{~T}$, the resistance increases with decreasing temperature below about $40 \mathrm{~K}$, and then forms a plateau, characteristic of semimetals with similar concentrations of different charge carriers. The bulk bands in $\mathrm{WAs}_{2}$ are presented in Figs. 4(c) and 4(d). Figure 4(c) shows bulk band without spin-orbit coupling. It does not indicate the presence of gapless Dirac-like dispersion. The inclusion of spin-orbit coupling [Fig. 4(d)] widens the gap between the linearly dispersing bands; our calculations are consistent with a previous study [32]. The Fermi surface of $\mathrm{WAs}_{2}$ contains multiple Fermi pockets suggesting a metallic nature of the material. The Fermi surface shows a dumbbellshaped feature with the middle of its handle located at the $\bar{\Gamma}$ point. In order to examine the Dirac state in $\mathrm{WAs}_{2}$, the dispersion maps were taken along the high-symmetry directions as shown in Figs. 4(f) and 4(g). Figure 4(f) shows the dispersion along the $\bar{Y}_{1}-\bar{X}-\bar{Y}_{1}$ direction as consisting of hole-like bands. The dispersion along the $\bar{Y}-\bar{\Gamma}-\bar{Y}$ direction also shows trivial bands with the band structure dominated by the bulk bands. In contrast to $\mathrm{MoAs}_{2}, \mathrm{WAs}_{2}$ does not possess a surface Diraclike dispersion in this direction.
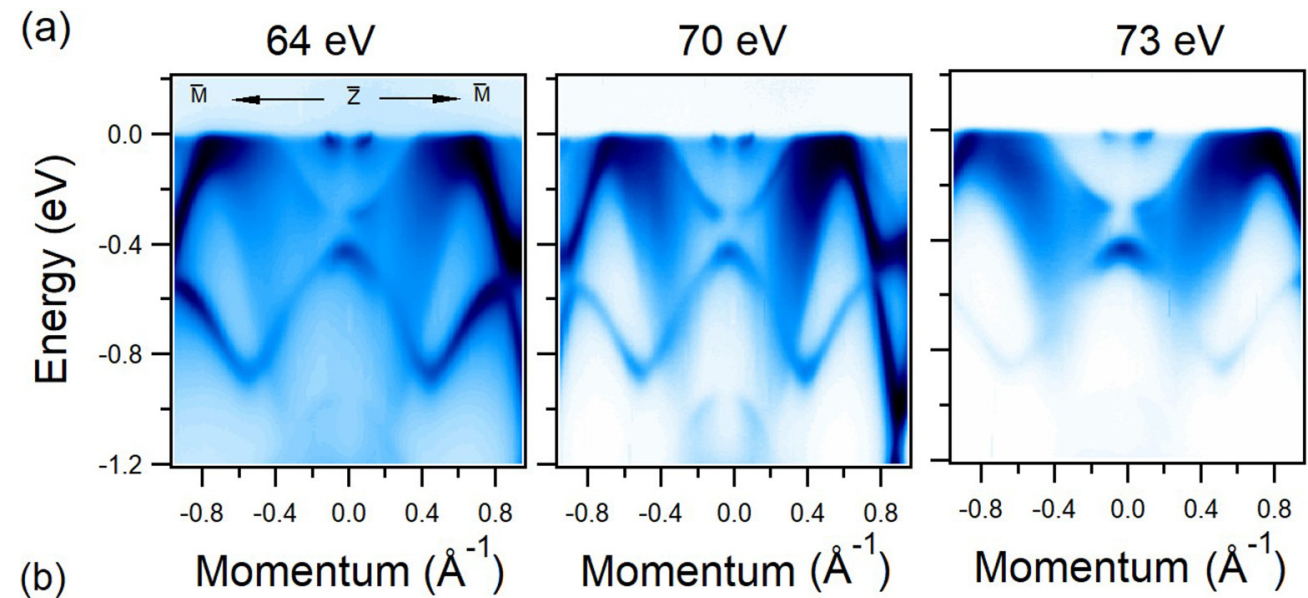

(b)
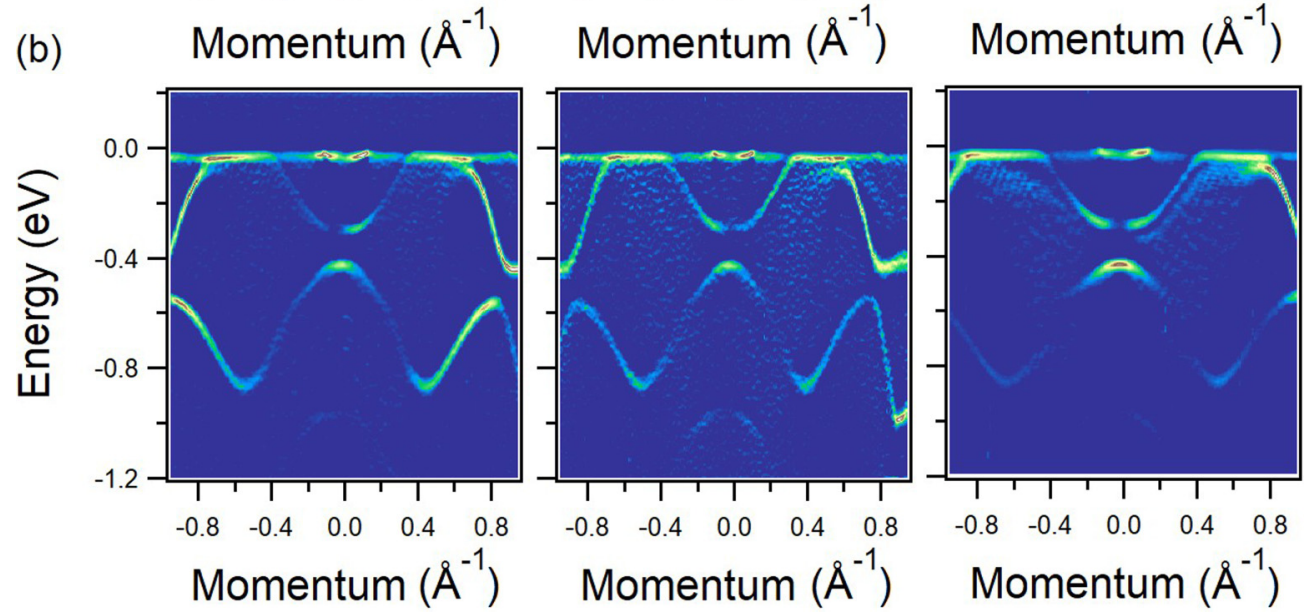

FIG. 10. Photon-energy-dependent electronic band dispersions along the $\bar{M}-\bar{Z}-\bar{M}$ direction from the (100) surface of MoAs $s_{2}$ (a) Experimental spectra at various indicated photon energies. (b) Second derivative plots corresponding to the spectra of panel (a). All the data were collected at the ALS beamline 10.0.1 at $15 \mathrm{~K}$. 


\section{CONCLUSIONS}

We have carried out a systematic ARPES study of transition-metal diarsenides $\mathrm{TAs}_{2}(\mathrm{~T}=\mathrm{Mo}, \mathrm{W})$ in conjunction with first-principles calculations. Surface electronic structures for the (001) and (100) surfaces are found to be quite different with both surfaces exhibiting the presence of multiple metallic Fermi surface pockets. Our symmetry analysis of the bulk band structures shows clearly that neither of these diarsenides supports a topological insulator state. This, along with the fact that these compounds respect time reversal as well as inversion symmetry, would seem to rule out their being either an intrinsic Dirac or a Weyl semimetal even though the band structures display some Dirac-like linearly-dispersing features. Our study highlights the importance of identifying the cleavage plane in electronic structure studies of low-symmetry systems.

\section{ACKNOWLEDGMENTS}

M.N. is supported by the Air Force Office of Scientific Research under Award No. FA9550-17-1-0415 and the National Science Foundation (NSF) CAREER Award No. DMR1847962. The work at Northeastern University was supported by the U.S. Department of Energy (DOE), Office of Science, Basic Energy Sciences Grant No. DE-FG02-07ER46352, and benefited from Northeastern University's Advanced Scientific Computation Center (ASCC) and the NERSC supercomput- ing center through DOE Grant No. DE-AC02-05CH11231. H.L. acknowledges the support by the Ministry of Science and Technology (MOST) in Taiwan under Grant No. MOST 109-2112-M-001-014-MY3. The work at TIFR Mumbai was supported by the Department of Atomic Energy of the Government of India under Project No. 12-R\&D-TFR-5.10-0100. This research used resources of the Advanced Light Source, a U.S. Department of Energy Office of Science User Facility, under Contract No. DE-AC02-05CH11231. We thank SungKwan Mo, Eli Rotenberg, Aaron Bostwick, and Chris Jozwiak for beamline assistance at the LBNL.

\section{APPENDIX A: EXPERIMENTAL GEOMETRY OF THE ARPES MEASUREMENTS}

The photoemission intensity $\left(\mathrm{I}_{0}\right)$ obtained in ARPES measurements is directly proportional to matrix element $\left.M_{f, i}^{k}\right|^{2}$ which, in turn, is proportional to $\left|\left\langle\phi_{f}^{k}|||\vec{\epsilon} . \vec{x}||| \phi_{i}^{k}\right\rangle\right|^{2}$, where $\phi_{i}^{k}$ and $\phi_{f}^{k}$ are the initial and final state wave functions of photoelectrons, respectively [44]. $\vec{\epsilon}$ and $\vec{x}$ denote the unit vector giving the direction of the electric field of the incoming photon and the unit position vector of electrons, respectively.

We present our experimental ARPES geometry in Fig. 5 showing that the incoming photon beam consists of a mixture of $s$ and p polarization. Both the analyzer slit and the incoming photons lie in the mirror plane defined by the analyzer slit and
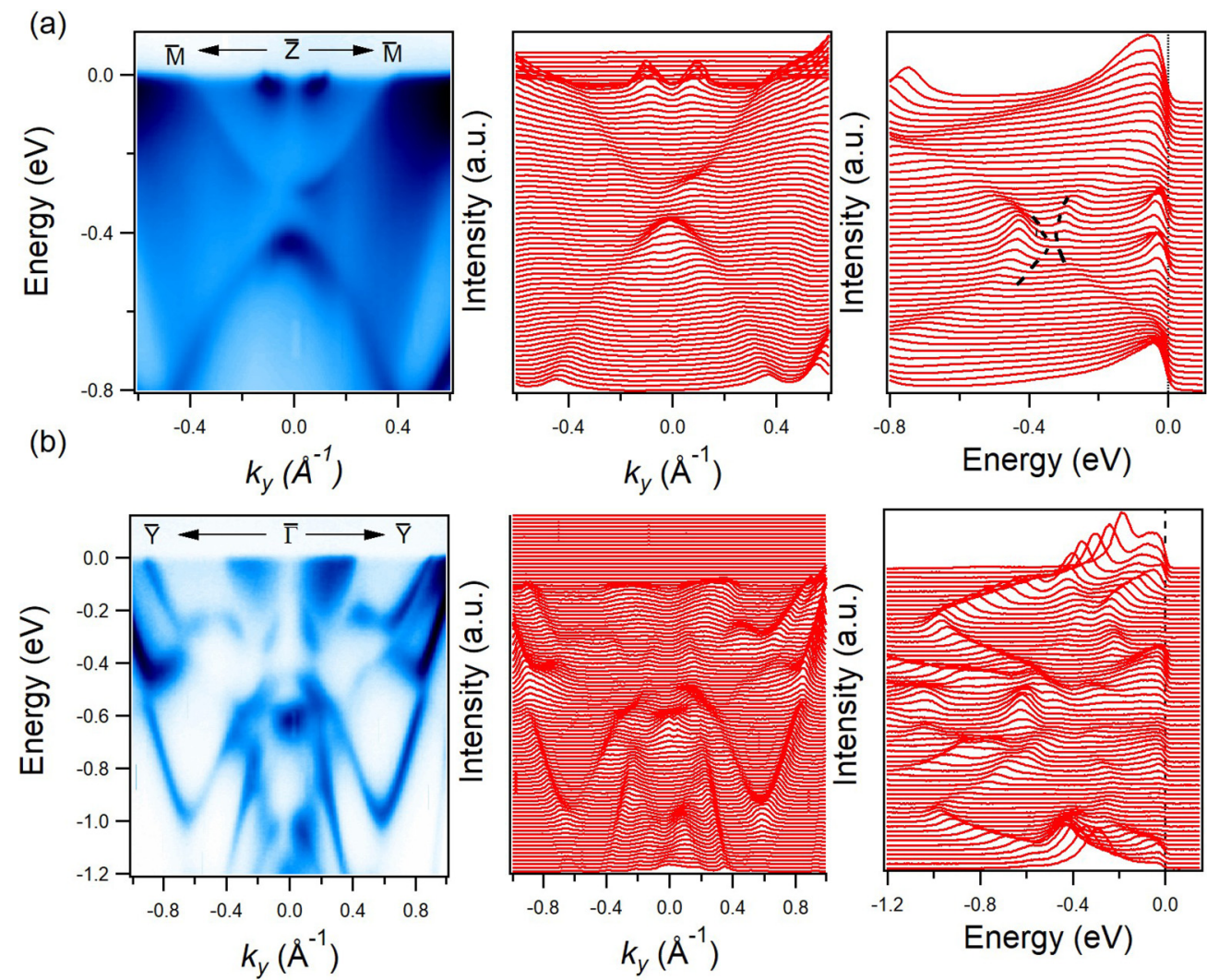

FIG. 11. Experimentally measured dispersion map, MDCs, and EDCs along the (a) $\bar{M}-\bar{Z}-\bar{M}$ and (b) $\bar{Y}-\bar{\Gamma}-\bar{Y}$ direction on the (100) surface of $\mathrm{MoAs}_{2}$. Data were collected at the ALS beamline 10.0.1 at $15 \mathrm{~K}$. 

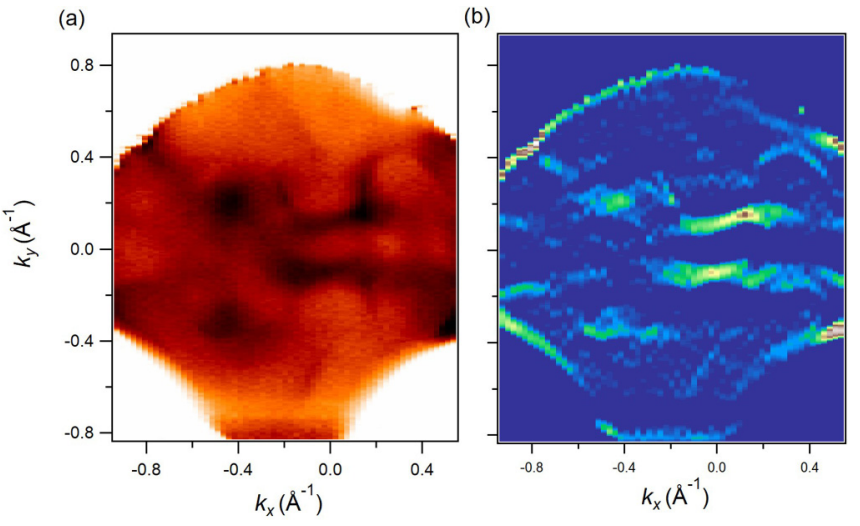

FIG. 12. Experimental Fermi surface map of $\mathrm{WAs}_{2}$ (a) from the (001) surface of $\mathrm{WAs}_{2}$ and (b) the corresponding second-derivative plot of the Fermi surface. The data were collected at the ALS beamline 7.0.2 at $20 \mathrm{~K}$ using $35 \mathrm{eV}$ photons.

the sample surface normal. In this setup $\vec{\epsilon} \cdot \vec{x}$ must be either odd or even to give a nonzero photo-intensity $[32,45]$.

\section{APPENDIX B: LEED MEASUREMENTS OF CLEAVED SURFACE OF MoAs 2}

In order to study the cleaved surface, we performed LEED and Laue spectroscopy measurements which are summarized in Fig. 6. Figures 6(a) and 6(b) show two different representative cleaving planes among multiple possible cleaving planes, LEED measurements demonstrate that $\mathrm{MoAs}_{2}$ does not have a preferred cleaving plane. Many samples were cleaved to inspect the preferred cleaving plane in $\mathrm{MoAs}_{2}$ which concluded aforementioned results. Laue diffraction pattern of natural flat surface is presented in Fig. 6(c). The lack of preferred cleaving plane in monoclonic transition metal diarsenides can be attributed to low crystal symmetry and absence of van der Waals interaction.

\section{APPENDIX C: ELECTRONIC STRUCTURES ON THE (001) SURFACE OF MoAs 2}

Figure 7 presents the bulk-band structure of $\mathrm{MoAs}_{2}$. The Fermi energy was artificially placed to lie between the bands 2 and 3. Our $\mathbb{Z}_{2}$ calculations for this band structure yield a trivial band structure. Figure 8 shows experimental photon-energy-dependent ARPES spectra for $\mathrm{MoAs}_{2}$ along the $\bar{Y}-\bar{\Gamma}-\bar{Y}$ direction and their second derivative plots. These data exhibit the presence of a gapless Dirac-like dispersion on the (001) plane. An electron-like band in the vicinity of the $\bar{\Gamma}$ point close to the Fermi level is suppressed at the photon energy of $92 \mathrm{eV}$. However $\alpha$-band [see Fig. 2(c)] does not disperse with photon energies indicating the surface state (see Fig. 8). In Fig. 9, we present MDCS and EDCs of dispersion map along the $\bar{Y}-\bar{\Gamma}-\bar{Y}$ direction which find the presence of gapless Dirac-like dispersion.

\section{APPENDIX D: ELECTRONIC STRUCTURES ON THE (100) SURFACE OF WAs ${ }_{2}$}

Figure 10 presents the photon-energy-dependent dispersion maps along the $\bar{M}-\bar{Z}-\bar{M}$ direction and corresponding second derivative plots. The dispersion maps at different photon energies demonstrate the absence of gapless Dirac-like state along this direction. MDCs and EDCs plots of dispersion maps along the $\bar{M}-\bar{Z}-\bar{M}$ and $\bar{M}-\bar{\Gamma}-\bar{M}$ (see Fig. 11) further confirm the absence of gapless Dirac-like state on the (100) plane.

\section{APPENDIX E: ELECTRONIC STRUCTURE OF THE (001) SURFACE OF WAs 2 .}

Figures 12 to 14 present electronic structures of $\mathrm{WAs}_{2}$. The Fermi surface is seen to be quite different from that of $\mathrm{MoAs}_{2}$. Photon-energy-dependent spectra along the $\bar{Y}-\bar{\Gamma}-\bar{Y}$ direction (see Fig. 13) indicate the presence of non-dispersive bands at the $\bar{\Gamma}$ point near the Fermi level. MDCs and EDCs along $\bar{Y}-\bar{\Gamma}-\bar{Y}$ and $\bar{Y}_{1}-\bar{X}-\bar{Y}_{1}$ are presented in Fig. 14 provide a clear indication of lack of Dirac states. The dispersion maps of $\mathrm{WAs}_{2}$ are quite different from those of $\mathrm{MoAs}_{2}$.
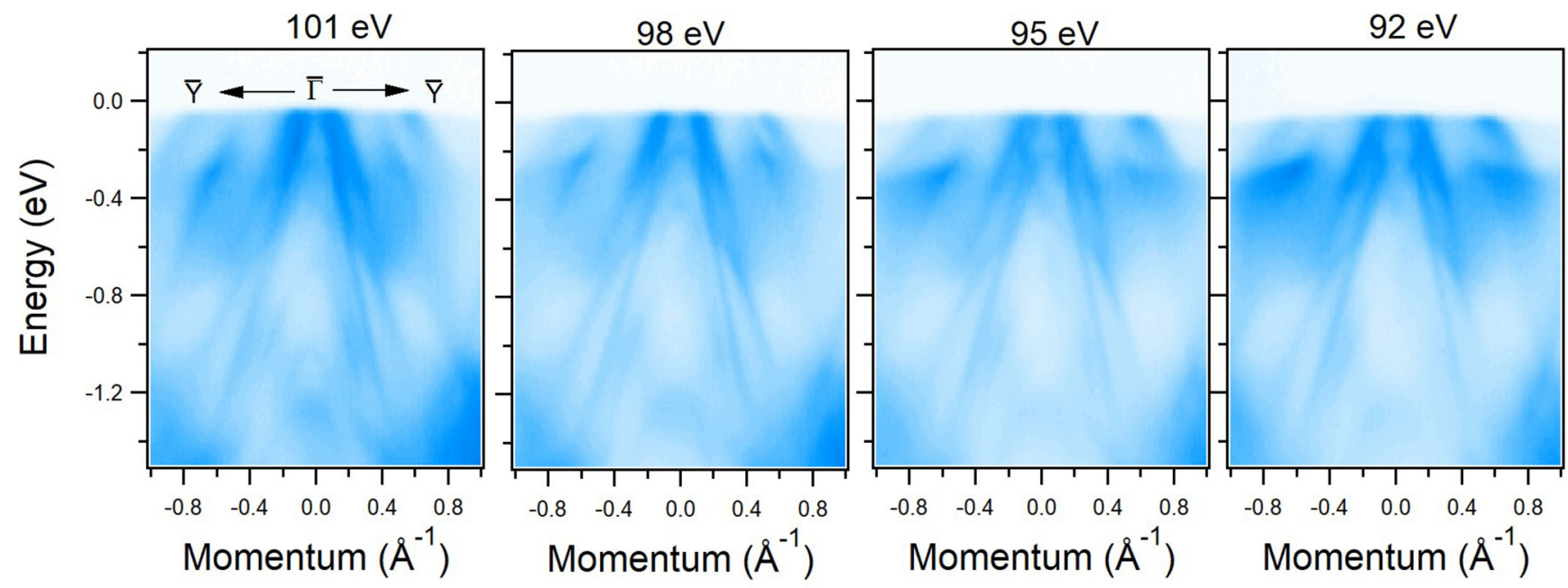

FIG. 13. Photon-energy-dependent spectral maps along the $\bar{Y}-\bar{\Gamma}-\bar{Y}$ direction on the (001) surface of WAs 2 . Data were collected at the ALS beamline 7.0.2. at $20 \mathrm{~K}$. 

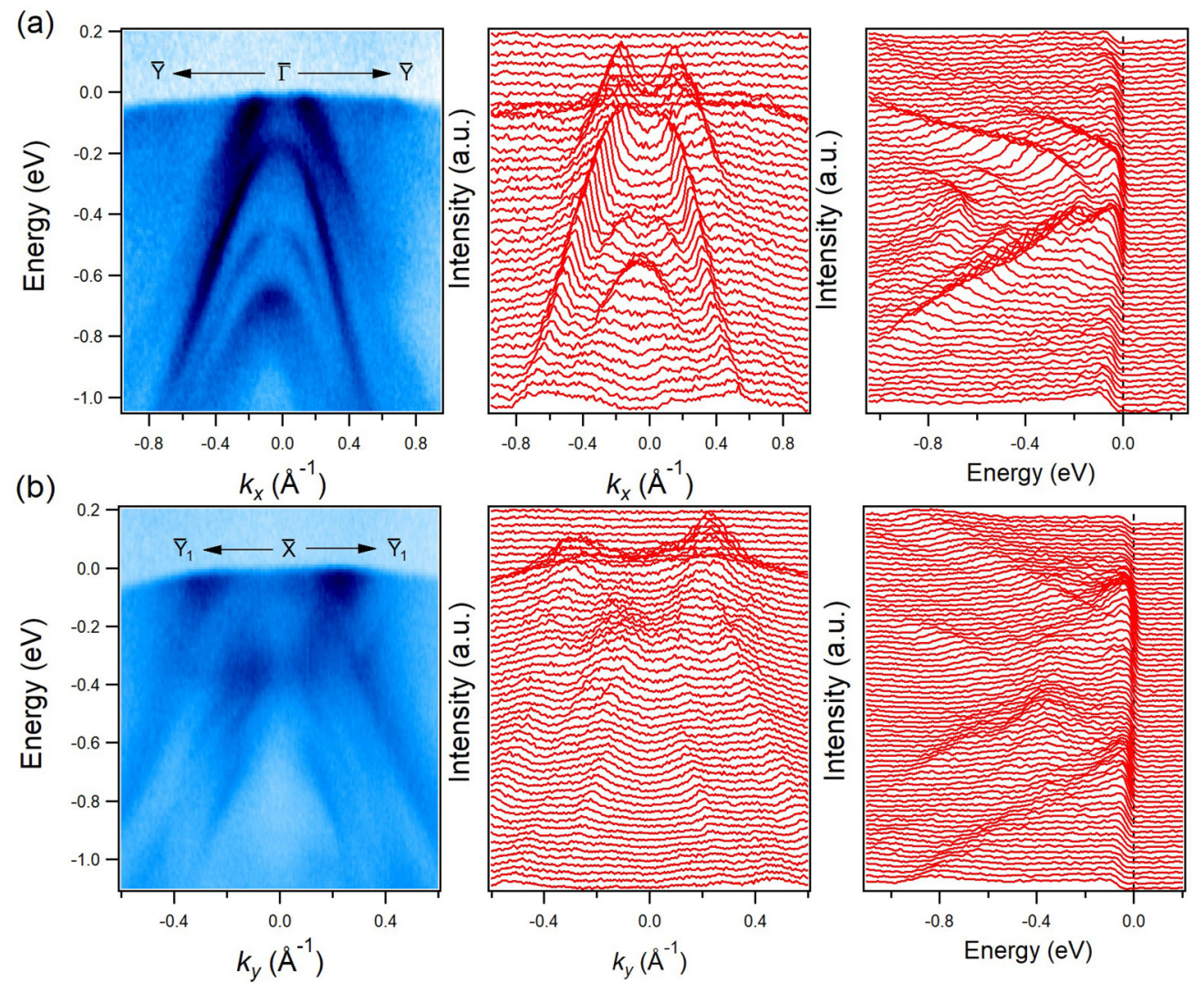

FIG. 14. Dispersion map, MDCs and EDCs along the (a) $\bar{Y}-\bar{\Gamma}-\bar{Y}$ direction, and the (b) $\bar{Y} 1-\bar{X}-\bar{Y}_{1}$ direction. Data were collected at the ALS beamline 7.0 .2 at $20 \mathrm{~K}$ using $35 \mathrm{eV}$ photons.

[1] M. Z. Hasan and C. L. Kane, Colloquium: Topological insulators, Rev. Mod. Phys. 82, 3045 (2010).

[2] J. E. Moore, The birth of topological insulators, Nature (London) 464, 194 (2010).

[3] X.-L. Qi, and S.-C. Zhang, Topological insulators and superconductors, Rev. Mod. Phys. 83, 1057 (2011).

[4] M. Z. Hasan, S.-Y. Xu, and M. Neupane, Topological Insulators, topological Dirac semimetals, topological crystalline insulators, and topological Kondo knsulators. in Topological Insulators: Fundamentals and Perspectives, edited by F. Ortmann, S. Roche, and S. O. Valenzuela (John Wiley \& Sons, New York, 2015).

[5] A. Bansil, H. Lin, and T. Das, Colloquium: Topological band theory, Rev. Mod. Phys. 88, 021004 (2016).

[6] S. Murakami, Phase transition between the quantum spin Hall and insulator phases in 3D: Emergence of a topological gapless phase, New J. Phys. 9, 356 (2007).

[7] S. M. Young, S. Zaheer, J. C. Y. Teo, C. L. Kane, E. J. Mele, and A. M. Rappe, Dirac Semimetal in Three Dimensions, Phys. Rev. Lett. 108, 140405 (2012).

[8] Z. Wang, H. Weng, Q. Wu, X. Dai, and Z. Fang, Threedimensional Dirac semimetal and quantum transport in $\mathrm{Cd}_{3} \mathrm{As}_{2}$, Phys. Rev. B 88, 125427 (2013).

[9] M. Neupane, S.-Y. Xu, R. Sankar, N. Alidoust, G. Bian, C. Liu, I. Belopolski, T.-R. Chang, H.-T. Jeng, H. Lin, A. Bansil, F. Chou, and M. Z. Hasan, Observation of a three-dimensional topological Dirac semimetal phase in high-mobility $\mathrm{Cd}_{3} \mathrm{As}_{2}$, Nat. Commun. 5, 3786 (2014).

[10] B.-J. Yang and N. Nagaosa, Classification of stable threedimensional Dirac semimetals with nontrivial topology, Nat. Commun. 5, 4898 (2014).

[11] M. Neupane, N. Alidoust, M. M. Hosen, J.-X. Zhu, K. Dimitri, S.-Y. Xu, N. Dhakal, R. Sankar, I. Belopolski, D. S. Sanchez, T.-R. Chang, H.-T. Jeng, K. Miyamoto, T. Okuda, H. Lin, A. Bansil, D. Kaczorowski, F. C. Chou, M. Z. Hasan, and T. Durakiewicz, Observation of the spin-polarized surface state in a noncentrosymmetric superconductor BiPd, Nat. Commun. 7, 13315 (2016).

[12] S. M. Young and C. L. Kane, Dirac Semimetals in Two Dimensions, Phys. Rev. Lett. 115, 126803 (2015).

[13] X. Wan, A. M. Turner, A. Vishwanath, and S. Savrasov, Topological semimetal and Fermi-arc surface states in the electronic structure of pyrochlore iridates, Phys. Rev. B 83, 205101 (2011).

[14] S.-M. Huang, S.-Y. Xu, I. Belopolski, C.-C. Lee, G. Chang, B. Wang, N. Alidoust, G. Bian, M. Neupane, C. Zhang, S. Jia, A. Bansil, H. Lin, and M. Z. Hasan, A Weyl Fermion semimetal with surface Fermi arcs in the transition metal monopnictide TaAs class, Nat. Commun. 6, 7373 (2015).

[15] H. Weng, C. Fang, Z. Fang, B. A. Bernevig, and X. Dai, Weyl Semimetal Phase in Noncentrosymmetric TransitionMetal Monophosphides, Phys. Rev. X 5, 011029 (2015). 
[16] S.-Y. Xu, I. Belopolski, N. Alidoust, M. Neupane, G. Bian, C. Zhang, R. Sankar, G. Chang, Z. Yuan, C.-C. Lee, S.-M. Huang, H. Zheng, J. Ma, D. S. Sanchez, B. Wang, A. Bansil, F. Chou, P. P. Shibayev, H. Lin, S. Jia et al., Discovery of a Weyl fermion semimetal and topological Fermi arcs, Science 349, 613 (2015).

[17] B. Q. Lv, H. M. Weng, B. B. Fu, X. P. Wang, H. Miao, J. Ma, P. Richard, X. C. Huang, L. X. Zhao, G. F. Chen, Z. Fang, X. Dai, T. Qian, and H. Ding, Experimental Discovery of Weyl Semimetal TaAs, Phys. Rev. X 5, 031013 (2015).

[18] I. Belopolski, S.-Y. Xu, D. S. Sanchez, G. Chang, C. Guo, M. Neupane, H. Zheng, C.-C. Lee, S.-M. Huang, G. Bian, N. Alidoust, T.-R. Chang, B. K. Wang, X. Zhang, A. Bansil, H.-T. Jeng, H. Lin, S. Jia, and M. Z. Hasan, Criteria for Directly Detecting Topological Fermi Arcs in Weyl Semimetals, Phys. Rev. Lett. 116, 066802 (2016).

[19] M. Z. Hasan, S.-Y. Xu, and G. Bian, Topological insulators, topological superconductors and Weyl fermion semimetals: discoveries, perspectives and outlooks, Phys. Scr. T164, 014001 (2015).

[20] G. Bian, T.-R. Chang, R. Sankar, S.-Y. Xu, H. Zheng, T. Neupert, C.-K. Chiu, S.-M. Huang, G. Chang, I. Belopolski, D. S. Sanchez, M. Neupane, N. Alidoust, C. Liu, B. K. Wang, C.-C. Lee, H.-T. Jeng, C. Zhang, Z. Yuan, S. Jia et al. Topological nodal-line fermions in spin-orbit metal $\mathrm{PbTaSe}_{2}$, Nat. Commun. 7, 10556 (2016).

[21] M. Neupane, I. Belopolski, M. M. Hosen, D. S. Sanchez, R. Sankar, M. Szlawska, S.-Y. Xu, K. Dimitri, N. Dhakal, P. Maldonado, P. M. Oppeneer, D. Kaczorowski, F. C. Chou, M. Z. Hasan, and T. Durakiewicz, Observation of topological nodal fermion semimetal phase in ZrSiS, Phys. Rev. B 93, 201104(R) (2016).

[22] M. M. Hosen, K. Dimitri, I. Belopolski, P. Maldonado, R. Sankar, N. Dhakal, G. Dhakal, T. Cole, P. M. Oppeneer, D. Kaczorowski, F. Chou, M. Z. Hasan, T. Durakiewicz, and M. Neupane, Tunability of the topological nodal-line semimetal phase in $\mathrm{ZrSi} X$-type materials $(X=\mathrm{S}, \mathrm{Se}, \mathrm{Te})$, Phys. Rev. B 95, 161101(R) (2017).

[23] C. Sims, M. M. Hosen, H. Aramberri, C.-Y. Huang, G. Dhakal, K. Dimitri, F. Kabir, S. Regmi, X. Zhou, T.-R. Chang, H. Lin, D. Kaczorowski, N. Kioussis, and M. Neupane, Terminationdependent topological surface states in nodal-loop semimetal $\mathrm{HfP}_{2}$, Phys. Rev. Materials 4, 054201 (2020).

[24] M. Z. Hasan, S.-Y. Xu, I. Belopolski, and S.- M. Huang, Discovery of Weyl Fermion Semimetals and Topological Fermi Arc States, Annu. Rev. Condens. Matter Phys. 8, 289 (2017).

[25] N. P. Armitage, E. J. Mele, and A. Vishwanath, Weyl and Dirac semimetals in three-dimensional solids, Rev. Mod. Phys. 90, 015001 (2018).

[26] A. A. Soluyanov, D. Gresch, Z. Wang, Q. Wu, M. Troyer, X. Dai, and B. A. Bernevig, Type-II Weyl semimetals, Prediction of Weyl semimetal in orthorhombic $\mathrm{MoTe}_{2}$, Nature (London) 527, 495 (2015).

[27] Y. Sun, S.-C. Wu, M. N. Ali, C. Felser, and B. Yan, Spectroscopic evidence for a type II Weyl semimetallic state in $\mathrm{MoTe}_{2}$, Phys. Rev. B 92, 161107(R) (2015).

[28] L. Huang, T. M. McCormick, M. Ochi, Z. Zhao, M.-t. Suzuki, R. Arita, Y. Wu, D. Mou, H. Cao, J. Yan, N. Tridevi, and A. Kaminski, Spectroscopic evidence for a type II Weyl semimetallic state in $\mathrm{MoTe}_{2}$, Nat. Mater. 15, 1155 (2016).
[29] Y. Wu, D. X. Mou, N. H. Jo, K. W. Sun, L. Huang, S. L. Budko, P. C. Canfield, and A. Kaminski, Observation of Fermi arcs in the type-II Weyl semimetal candidate $\mathrm{WTe}_{2}$, Phys. Rev. B 94, 121113(R) (2016).

[30] G. Autès, D. Gresch, M. Troyer, A. A. Soluyanov, and O. V. Yazyev, Robust Type-II Weyl Semimetal Phase in Transition Metal Diphosphides $X \mathrm{P}_{2}(X=\mathrm{Mo}, \mathrm{W})$, Phys. Rev. Lett. 117, 066402 (2016).

[31] J. Wang, L. Li, W. You, T. Wang, C. Cao, J. Dai and Y. Li, Magnetoresistance and robust resistivity plateau in $\mathrm{MoAs}_{2}$, Sci. Rep. 7, 15669 (2017).

[32] J. Chen, Y.-K. Li, J. Dai, and C. Cao, Electronic structure and topological properties of centrosymmetric $\mathrm{MoAs}_{2} / \mathrm{WAs}_{2}$ from first principles, Sci. Rep. 7, 10491 (2017).

[33] R. Lou, Y. F. Xu, L.-X. Zhao, Z.-Q. Han, P.-J. Guo, M. Li, J.-C. Wang, B.-B. Fu, Z.-H. Liu, Y.-B. Huang, P. Richard, T. Qian, K. Liu, G.-F. Chen, H. M. Weng, H. Ding, and S.-C. Wang, Observation of open-orbit Fermi surface topology in the extremely large magnetoresistance semimetal $\mathrm{MoAs}_{2}$, Phys. Rev. B 96, 241106(R) (2017).

[34] R. Singha, A. Pariari, G. K. Gupta, T. Das, and P. Mandal, Probing the Fermi surface and magnetotransport properties of $\mathrm{MoAs}_{2}$, Phys. Rev. B 97, 155120 (2018).

[35] J. J. Murray, J. B. Taylor, and L. Usner, Halogen transport of molybdenum arsenides and other transition metal pnictides, J. Cryst. Growth 15, 231 (1972).

[36] G. Kresse and D. Joubert, From ultrasoft pseudopotentials to the projector augmented-wave method, Phys. Rev. B 59, 1758 (1999).

[37] P. Hohenberg and W. Kohn, Inhomogeneous electron gas, Phys. Rev. 136, B864 (1964).

[38] G. Kresse and J. Furthmuller, Efficient iterative schemes for ab initio total-energy calculations using a plane-wave basis set, Phys. Rev. B 54, 11169 (1996).

[39] J. P. Perdew, K. Burke, and M. Ernzerhof, Generalized Gradient Approximation Made Simple, Phys. Rev. Lett. 77, 3865 (1996).

[40] N. Marzari and D. Vanderbilt, Maximally localized generalized Wannier functions for composite energy bands, Phys. Rev. B 56, 12847 (1997).

[41] Q. Wu, S. Zhang, H.-F. Song, M. Troyer, and A. A. Soluyanov, WannierTools: An open-source software package for novel topological materials, Comput. Phys. Commun. 224, 405 (2018).

[42] A. Bansil and M. Lindroos, Importance of Matrix Elements in the ARPES Spectra of BISCO, Phys. Rev. Lett. 83, 5154 (1999).

[43] M. R. Scholz, J. Sanchez-Barriga, J. Braun, D. Marchenko, A. Varykhalov, M. Lindroos, Y. J. Wang, H. Lin, A. Bansil, J. Minr, H. Ebert, A. Volykhov, L. V. Yashina, and O. Rader, Reversal of the Circular Dichroism in Angle-Resolved Photoemission from $\mathrm{Bi}_{2} \mathrm{Te}_{3}$, Phys. Rev. Lett. 110, 216801 (2013).

[44] A. Damascelli, Z. Hussain, and Z.-X. Shen, Angle-resolved photoemission studies of the cuprate superconductors, Rev. Mod. Phys. 75, 473 (2003).

[45] S. Tang, C. Zhang, D. Wong, Z. Pedramrazi, H.-Z. Tsai, C. Jia, B. Moritz, M. Claassen, H. Ryu, S. Kahn, J. Jiang, H. Yan, M. Hashimoto, D. Lu, R. G. Moore, C.-C. Hwang, C. Hwang, Z. Hussain, Y. Chen, M. M. Ugeda et al., Quantum spin Hall state in monolayer 1T'-WTe 2 , Nat. Phys. 13, 683 (2017). 